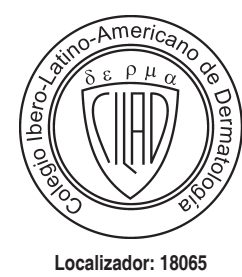

doi: $10.35366 / 91756$

\title{
Queloides auriculares: una nueva opción de tratamiento
}

\author{
Auricular keloids: a new treatment option
}

Karhyn Milagros Ríos-Serna, * Diego del Ojo-Cordero

Palabras clave: Cicatriz queloidea, pabellón auricular, láser neodimio.

Keywords: Keloid scar, auricle, neodymium laser.

* Dermatóloga del servicio de Dermatología del Hospital Guillermo Kaelin de la Fuente. Lima-Perú.

¿Dermatólogo del servicio de Dermatología. Hospital Puerto Real. Cádiz-España.

Conflicto de intereses: Ninguno.

Recibido:

21/Octubre/2018.

Aceptado:

11/Junio/2019.

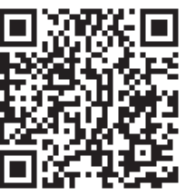

\section{RESUMEN}

Introducción: El pabellón auricular es una localización de riesgo para la aparición de queloides $(\mathrm{Q})$, ya que es una de las patologías de más difícil tratamiento por el alto porcentaje de recidivas que presentan, hasta $100 \%$ en tratamientos únicos y $50 \%$ en tratamientos combinados. Objetivo: Nuestro objetivo es revisar nuestra experiencia y plantear un posible tratamiento de cicatrices queloideas en el pabellón auricular. Material y métodos: Hemos revisado queloides auriculares (QA) en los últimos 10 años; se ha recogido la epidemiología, la etiología de la herida, el tratamiento y los resultados. Resultados: De los 23 pacientes y los 28 pabellones auriculares afectados por queloides, la edad más frecuente fue entre 20 a 29 años. El 65\% fueron mujeres, $80 \%$ de fototipo III; $53.5 \%$ de los QA afectaban a la oreja derecha, $56 \%$ se produjeron en el lóbulo, 28\% en el hélix y 16\% fueron retroauriculares. En $73.9 \%$ la causa fue piercing y el uso de pendientes, y en $17.4 \%$ fue un antecedente quirúrgico. Del total, $34.8 \%$ realizaron tratamientos previos y $65.2 \%$ no realizó ningún tratamiento. El tratamiento fue infiltración con triamcinolona (tres sesiones), seguido de láser Neodimio Yag (tres sesiones) y al finalizar láser CO2. Después se mantuvieron con presoterapia por un año para disminuir las probabilidades de recidiva. En 100\% de los pacientes no se encontró recidiva. Conclusiones: Dentro de este estudio hemos demostrado la seguridad y la eficacia de una combinación de terapias para los queloides auriculares y proponemos una posible terapia exitosa.

\section{ABStRACT}

Introduction: The auricle is a risk location for the appearance of keloids $(K)$ and is one of the most difficult pathologies to treat due to the high percentage of recurrences, which show up to $100 \%$ in single treatments and $50 \%$ in combined treatments. Objective: Our objective is to review our experience and to propose a possible treatment of keloid scars in the auricle. Material and methods: We have seen auricular keloids (AK) over the past 10 years, collecting epidemiology, wound etiology, treatment and outcomes. Results: Of the 23 patients and 28 auricles affected by keloids, the most frequent age was between 20 to 29 years. $65 \%$ were women, $80 \%$ of whom were phototype III; $53.5 \%$ of AK affected the right ear, AK occurred $56 \%$ of the time in the lobe, $28 \%$ in the helix and $16 \%$ of $A K$ were retroauricular. $73.9 \%$ of the time the cause was piercing and use of earrings and $17.4 \%$ of patients had a surgical background. Of the total number of patients, $34.8 \%$ had previous treatments and $65.2 \%$ did not undergo any treatment. The treatment was infiltration of triamcinolone (three sessions), followed by Neodimio Yag laser (three sessions) and finally CO2 laser. It was subsequently maintained with presotherapy for one year to decrease the chances of relapse. No recurrence was found in any of the patients. Conclusions: In this study we have demonstrated the safety and efficacy of a combination of therapies for auricular keloids and propose a possible successful therapy.

\section{INTRODUCCIÓN}

L a cicatriz queloidea o queloide $(\mathrm{Q})$ es una patología o desorden fibroproliferativo de la dermis, se presenta sólo en humanos y ocurre como resultado de una respuesta regenerativa anómala ante una lesión de la piel. ${ }^{1-4}$

Se caracteriza por presentar sobreelevación de la piel, cambios de coloración y por extenderse más allá de los límites de la lesión inicial. Esta última propiedad la distingue de las cicatrices hipertróficas, otra forma de crecimiento exacerbado de los tejidos cicatriciales. ${ }^{5-8}$
Este proceso de reparación atraviesa diferentes fases o etapas que ocurren en forma simultánea y se influyen mutuamente, de manera que las alteraciones en una etapa pueden beneficiar o perjudicar a las otras. ${ }^{6}$ No hay una hipótesis única que explique el proceso de forma adecuada, pero se le han atribuido a la formación del queloide las alteraciones en la regulación de los factores de crecimiento, el recambio aberrante de colágena (el mecanismo por el cual se ve aumentada la cantidad de colágeno no es por un exceso en el número de fibroblastos, sino por un aumento en la 
producción de proteínas de matriz y una disminución de la degradación de estas proteínas), los factores genéticos, la disfunción inmunológica, la reacción al sebo y la alteraciones mecánicas. ${ }^{3,4}$

No existe ninguna relación causa-efecto demostrada para la aparición del Q, por lo que su formación es imprevisible, pero sí se han encontrado algunas condiciones que lo favorecen: localización (predominan sobre esternón, deltoides y pabellón auricular) y características del paciente (afecta a cualquier etnia, aunque la piel oscura se ve afectada 15 veces más que la tez clara, ${ }^{7}$ en situaciones de hipersecreción pituitaria como el embarazo y la adolescencia, y antecedentes como presencia de Q en otra localización o antecedentes familiares de Q). ${ }^{2,5}$ Además los queloides se presentan en áreas donde la concentración de melanocitos es mayor, por lo tanto, son raros en las palmas de las manos y las plantas de los pies, donde la concentración melanítica es menor. ${ }^{4,6}$

Se presentan por igual en ambos sexos y son más frecuentes durante las primeras décadas de la vida; ${ }^{5}$ se ha determinado que la cicatrización queloide ocurre entre los 10 y los 30 años de edad, es rara en personas más jóvenes y en los mayores de 50 años. ${ }^{6,8}$ Hay tendencia familiar y asociación genética con el HLA-B14, B21, Bw16, DR5, DQw3 y con el grupo sanguíneo tipo A; así como, asociación con enfermedades inflamatorias como acné conglobata, varicela o reacciones a cuerpo extraño.,

El pabellón auricular es una localización de riesgo y el factor predisponente más común para la aparición de Q. ${ }^{6}$ Los queloides auriculares se han asociado al empleo de aros, piercing, traumatismos, quemaduras y cirugías, y su incidencia es creciente. ${ }^{2}$

El tratamiento es muy complicado, y cuando se realiza existe la tendencia a la recidiva, rara vez con remisión total, y aunque existe una amplia variedad de tratamientos ninguno es efectivo en todos los casos; sin embargo, hay la probabilidad de que lo más efectivo sea la combinación de varios tratamientos. ${ }^{5}$

Se han descrito numerosas alternativas terapéuticas; entre los tratamientos no quirúrgicos los más empleados incluyen la compresión o presoterapia. En la reparación del defecto auricular se ha empleado el cierre primario, la cicatrización por segunda intención, diferentes tipos de colgajos locales e injertos de piel, enucleación del volumen principal de fibrosis con plastia del remanente cutáneo (fillet flap), los corticoides intralesionales, el empleo de láminas de silicona y la radioterapia. ${ }^{5}$

La administración intralesional y tópica de agentes quimioterapéuticos (como la mitomicina C) representa un complemento común a la cirugía como modalidad de tratamiento primario. El 5-fluorouracilo así como el Imiquimod representan una terapia tópica adicional que se utiliza para atenuar la recurrencia de queloides después de la resección, las cuales trabajan como un modulador de respuesta inmune. Sin embargo, se evidencia una tasa de recurrencia de $4 \%$ con la complicación más común: necrosis de tejido local. ${ }^{9}$ Otras alternativas mencionadas en la literatura son el interferón alfa, crioterapia y láser, entre otras. ${ }^{5}$

El objetivo del presente trabajo es revisar nuestra experiencia de 10 años en el tratamiento de queloides auriculares, ya que se trata de una patología muy frustrante para el especialista que no puede saber si su paciente, aun siendo perfectamente tratado, sufrirá la recidiva de la lesión; además se conoce la existencia de múltiples terapias sin ningún resultado claro, ni satisfactorio y que evite las recidivas que son el principal inconveniente. Por tanto, en el presente trabajo aportamos una posible terapéutica para el manejo de estas lesiones.

\section{MATERIAL Y MÉTODOS}

Se ha revisado a través de un diseño de estudio retrospectivo todos los casos de QA tratados en el Hospital Puerto Real-España en los últimos 10 años. Se registró en una hoja de recolección de datos, confeccionada por los autores, los datos personales (edad, sexo, raza, antecedentes personales), demográficos (procedencia), el mecanismo de la herida inicial (otoplastia, exéresis, piercing, etcétera), localización de la lesión (hélix, lóbulo, antihélix, retroauricular/ uni- o bilateral), los tratamientos efectuados y su evolución, las recidivas y su manejo.

Esta hoja fue llenada a través de la revisión de las historias clínicas de los pacientes atendidos por queloides auriculares. Para el análisis de las características anteriormente mencionadas se utilizaron algunos indicadores de resumen como tasas y proporciones.

Hemos revisado la literatura al respecto para conocer las nuevas opciones terapéuticas.

En el tratamiento empleado se aplicó mepivacaína $2 \%$ a partes iguales con triamcinolona intralesional (40 $\mathrm{mg} / \mathrm{ml}$ ) en las cicatrices, en tres ocasiones con diferencia de cuatro semanas entre aplicación, y seguido en cada sesión por el Láser Neodimio YAG con una X: 1,064 nm, fluencia $120 \mathrm{~J} / \mathrm{cm}$, duración de pulso: $10 \mathrm{~ms}$, spot: 3-10 dependiendo del tamaño.

A la cuarta sesión se aplicó sólo el Láser Neodimio YAG con similares parámetros y a la quinta sesión Láser $\mathrm{CO} 2$ para eliminar el tejido residual. Posteriormente se mantuvo con presoterapia (con un pendiente de 
presión) por un año para disminuir las probabilidades de recidiva.

Se les realizó controles cada año, siendo el seguimiento durante 10 años y en su evolución no se evidenció recidivas hasta la actualidad.

Algunas de las cicatrices fueron enviadas a patología para su estudio para confirmar el diagnóstico (Figura 1).

\section{RESULTADOS}

En los últimos 10 años se ha tratado 23 QA en pacientes entre 19 y 78 años (39\% comprende rangos de edad entre 20 a 29 años, y $21.7 \%$ de 30 a 39 años) al inicio de la lesión. El $65 \%$ fueron mujeres, $80 \%$ de fototipo III, $15 \%$ IV y $5 \%$ II. No hubo antecedentes personales de importancia. El $53.5 \%$ de los QA afectaban a la oreja derecha $(21.7 \%$ fueron bilaterales).

Catorce de las lesiones (56\%) se produjeron en el lóbulo, siete en el hélix (28\%), y cuatro retroauriculares (16\%). En 17 casos (73.9\%) la lesión inicial se produjo por fines estéticos (piercing y uso de pendientes). En cuatro casos (17.4\%) existe un antecedente quirúrgico: en un caso la otoplastia, en dos una biopsia/exéresis de CA auricular, uno con herida accidental que se suturó; y dos casos (8.7\%) como secuelas de acné.

Los años de evolución con el queloide fue en $25 \%$ de cinco años, $37.5 \%$ durante un año y $12.5 \%$ durante dos, tres y cuatro años cada uno.

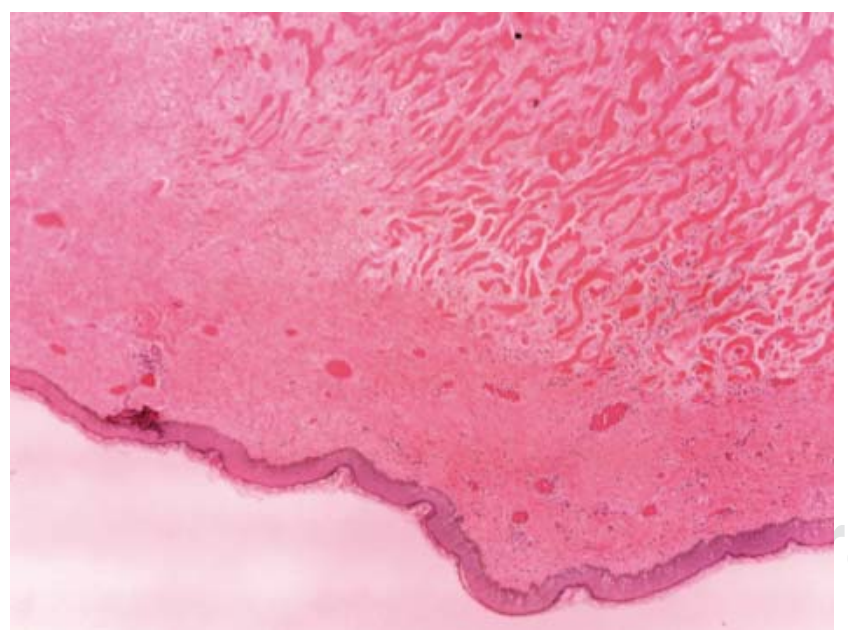

Figura 1: Biopsia: dermis superficial reducida a una pequeña banda hialina. Dermis profunda con números haces de fibras colágeno y fibroblastos que se entrecruzan, conformando bandas gruesas de colágeno hialinizado. (Hematoxilina-eosina 200X).

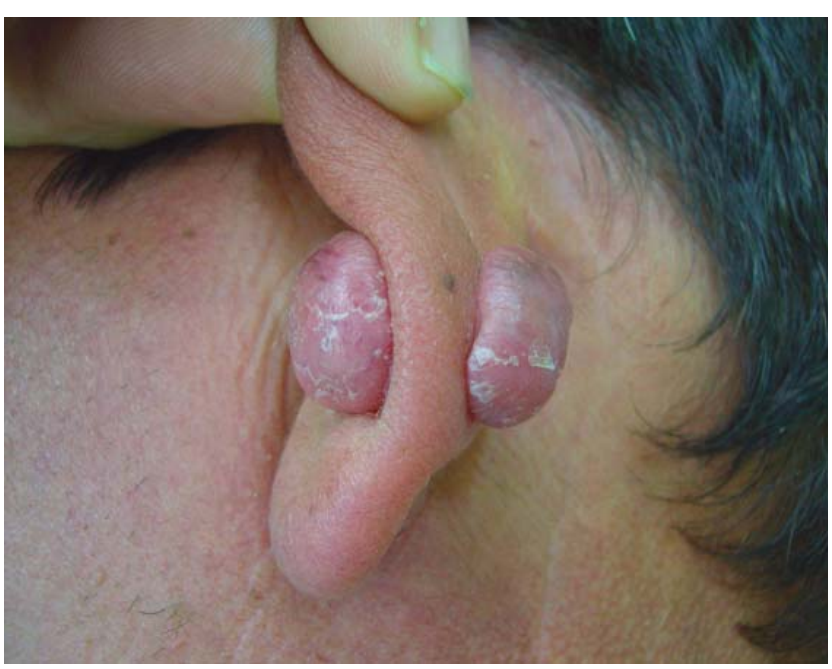

Figura 2: Cicatriz queloide tipo tumor que atraviesa y compromete ambas caras de pabellón auricular izquierdo. Pretratamiento.

Realizaron tratamientos previos $34.8 \%$, siendo el $17.4 \%$ cirugía entre tres y cuatro ocasiones cada uno, 8.8\% utilizaron parches, $4.3 \%$ láser $\mathrm{CO} 2$ y $4.3 \%$ con cremas corticoides, y $65.2 \%$ no realizó ningún tratamiento con anterioridad.

El tratamiento fue infiltración, seguido de láser Neodimio Yag y láser CO2 en los 23 casos (100\%), después se mantuvo con presoterapia por un año. No se encontró recidiva en ninguno de ellos (Figuras 2 a 5).

\section{DISCUSIÓN}

Entre las muchas áreas en el cuerpo humano que son propensas a la formación de queloides, las orejas han sido documentadas para tener una de las más altas tasas de recurrencia. Aparte de sus atributos comunes de dolor y picazón, lo que los distingue es su posible influencia en la imagen corporal, debido a que no están naturalmente destinadas a ser cubiertas, estas lesiones pueden afectar gravemente a la autoimagen física del paciente. ${ }^{2}$

Los resultados obtenidos en cuanto a las características del grupo (sexo, etiología, localización) son similares a los de otras series. ${ }^{2}$ En la mayoría de los casos, el grupo afectado son mujeres jóvenes quienes son comprensiblemente sensibles a las anormalidades cosméticas. ${ }^{10}$

En cuanto a la edad, el rango de edad con mayor proporción encontrada es la comprendida entre 20 y 29 años con $39.1 \%$, seguida de $21.7 \%$ entre los 30 y 39 años, alcanzando más de $60 \%$ ambos. Esto coincide con las demás series en que lo más habitual es que afecte entre 
los 11 y los 30 años y es rara en personas con edades más extremas de la vida.

En fototipos, el III fue el que más casos se presentaron en comparación con el II, ya que es el tipo más frecuente en esta población y como se mencionó en estudios anteriores, las personas con piel oscura parecen ser más propensas a la formación de queloides, la razón exacta es desconocida; sin embargo, una posible etiología genética y familiar relacionada con la excesiva actividad de los fibroblastos ha sido postulada por los investigadores. ${ }^{10}$

Un tercio de nuestros pacientes antes de tratarlos ya habían probado otros métodos. Entre los encontrados están los parches de silicona sobre la cicatriz, se cree que favorecen la correcta cicatrización por el aumento de la hidratación de los tejidos, aunque es difícil su aplicación sobre los relieves auriculares y del rostro. ${ }^{2}$

Otro tratamiento utilizado previamente fue el quirúrgiCo, si es aislado, conlleva un altísimo riesgo de recidiva de la lesión, de entre 55 y 100\% de los casos según las series, incluso en un muy corto periodo de tiempo. ${ }^{2} \mathrm{~A}$ veces, la simple escisión total de un queloide estimula una síntesis de colágeno adicional, lo que provoca una recurrencia rápida de un queloide aún mayor que el queloide inicial. La escisión quirúrgica de un queloide solo está asociada con una alta tasa de recidiva, y por lo tanto, debe combinarse con terapia adyuvante. ${ }^{11}$

La escisión con láser de CO2 también se utilizó previamente, tiene un porcentaje muy similar de recidiva de $50 \%$ si se compara con una escisión simple. Lo que

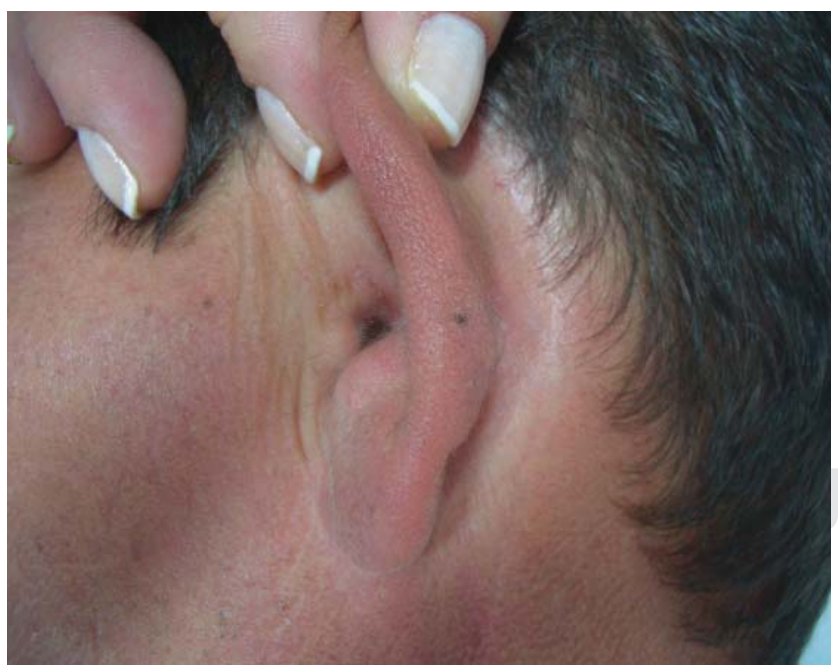

Figura 3: Paciente después de realizar tratamiento, no se evidencia ninguna lesión.

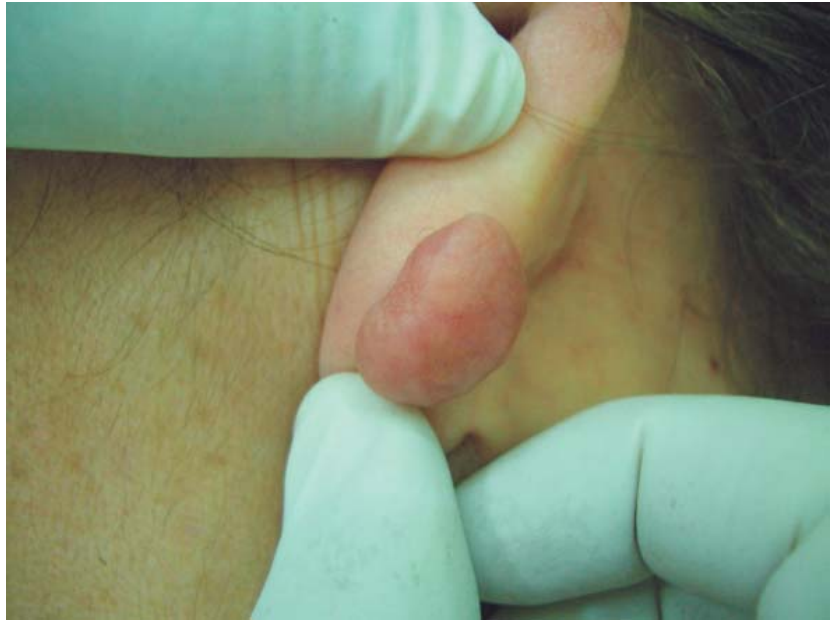

Figura 4: Cicatriz queloide que compromete cara posterior de pabellón auricular izquierdo. Pretratamiento.

se está utilizando es la combinación del láser $\mathrm{CO} 2$ con otras modalidades como lo veremos en nuestros casos. ${ }^{1}$

El tratamiento de queloides auriculares ha sido históricamente subóptimo, se caracteriza por malestar, mala respuesta y altas tasas de recurrencia. Una serie de modalidades han sido probadas a través de los años y la búsqueda de la «modalidad perfecta» continúa. ${ }^{10}$

Al revisar la bibliografía sobre la combinación de tratamientos encontramos a Bejarano y su grupo, que de 11 queloides auriculares estudiados en los últimos 10 años, dos mejoraron con corticoides tópicos. El tratamiento fue quirúrgico en nueve casos, cuatro de ellos con injerto: cinco se curaron y cuatro tuvieron recidiva. ${ }^{2}$

Gutiérrez y colegas hicieron una revisión en los últimos seis años con terapia combinada en 51 pacientes y 64 pabellones auriculares afectados por queloides. Aplicaron de forma preoperatoria tres dosis de triamcinolona o betametasona intralesional, con diferencia de cuatro a seis semanas entre dosis, y cuatro semanas después de la última dosis realizaron resección de la cicatriz. De las 48 cicatrices que recibieron tratamiento quirúrgico hubo recidiva en $12.5 \%$ (seis cicatrices), con un seguimiento de entre ocho meses a seis años. ${ }^{3}$

Castillo y colaboradores emplearon cirugía y compresión, con un tiempo de seguimiento medio de 18 meses, no hubo recidivas en los 10 queloides de los lóbulos, pero si una recidiva del queloide del tercio superior de la oreja, lo que representa $7.6 \%$ de los casos. ${ }^{5}$

Zulqarnain y su equipo trataron 24 queloides auriculares gigantes (tamaño medio, $11 \mathrm{~cm}^{2}$ ) entre enero de 2008 y julio de 2012, utilizaron un nuevo protocolo consistente 
en una escisión completa, injerto de piel, una inyección intraoperatoria de una hora de triamcinolona, radioterapia inmediata y terapia de presión sostenida. A un año, la tasa de éxito fue de $87.5 \%$ y la de recurrencia fue de $12.5 \% .{ }^{10}$

No se encontraron revisiones que hayan utilizado la misma terapéutica de nosotros y que no haya recurrencias después de 10 años.

Utilizamos en el tratamiento corticoides intralesionales, siendo el compuesto más utilizado la triamcinolona. ${ }^{2}$ La triamcinolona inhibe la proliferación de fibroblastos normales y queloides, inhibe la síntesis de colágeno, aumenta la producción de colagenasa y reduce los niveles de inhibidores de ésta. Además, se inducen cambios ultraestructurales en la síntesis de colágeno, que mejoran la organización de los haces de colágeno y degeneran los nódulos de colágeno queloideo característicos. No se ha demostrado ninguna ventaja clara para un tipo específico de corticosteroide. ${ }^{12,13}$

Aunque la administración intralesional de corticosteroides presenta tasas de respuesta altamente variables de 50 a 100\%, también se acompaña de tasas de recurrencia de 9 a 50\%, es por ello que se prefirió asociar el Láser de Neodimio YAG en modo pulsado (pulsos de milisegundos) posterior a la infiltración. ${ }^{10}$ Un factor muy importante para el desarrollo y mantenimiento de los queloides es la vascularización, por lo tanto se utilizó el Láser de Neodimio YAG con este fin, ya que es utilizado para el tratamiento de lesiones vasculares profundas. ${ }^{14-16}$

El láser CO2 es otra terapia adyuvante de la exéresis, este láser emite una luz de 10,600 nm que se encuentra dentro del espectro infrarrojo, y en la piel éste será absor-

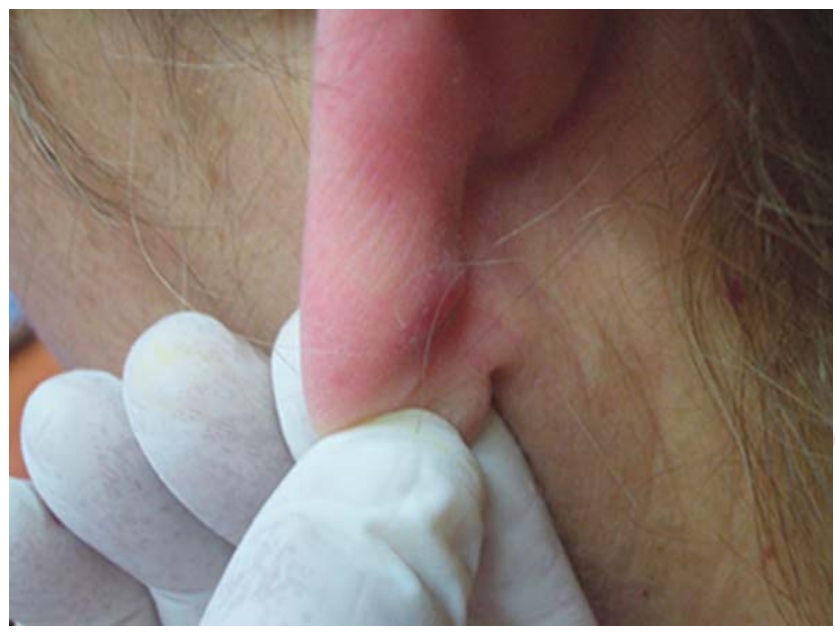

Figura 5: Paciente después de realizar tratamiento, no se evidencia ninguna lesión. bido por el agua. Se produce entonces un calentamiento importante del agua intracelular que lleva a la destrucción celular; dada la alta cantidad de agua que presentan todos los tejidos éste será absorbido en cuanto toca la superficie cutánea, produciendo una ablación o destrucción de la epidermis. El láser pretende con estos sistemas ablativos eliminar la lesión existente (por una parte logra un aplanamiento de la cicatriz), pero no actúa sobre los mecanismos subyacentes, por lo que en la mayoría de los casos se volverá a formar la lesión si se trata en monoterapia. Por ello, en caso de utilizar sistemas ablativos, debemos asociarlos con otro tipo de tratamiento que intente conseguir estabilizar estas lesiones, tal como se utilizó en nuestros pacientes. ${ }^{16}$

Como paso final en nuestro tratamiento se realizó presoterapia, la cual tiene un mecanismo de acción multifactorial, no definitivamente aclarado, pero la presión causa hipoxia localizada, lo que resulta en degeneración y desintegración de fibroblastos, debido a la disminución de la cohesión del colágeno y al aumento de la actividad colagenasa. El problema principal de esta terapia es la adhesión al tratamiento, ya que los dispositivos que aplican la presión deben colocarse hasta seis meses para prevenir una recidiva. ${ }^{2,12}$ Debido a la anatomía compleja y altamente sensible de la aurícula, la terapia de compresión está limitada por la alineación óptima del dispositivo y la suficiente presión a largo plazo. La presión ejercida debe ser de al menos $24 \mathrm{mmHg}$ para exceder la presión capilar inherente, pero debe permanecer por debajo de 30 $\mathrm{mmHg}$; de lo contrario, la circulación sanguínea periférica disminuye, resultando en necrosis tisular. ${ }^{12}$

\section{CONCLUSIONES}

Podemos concluir que el escaso conocimiento de la patogénesis de los queloides se refleja en la multitud de enfoques terapéuticos. Hasta entonces tenemos que continuar nuestra búsqueda para el establecimiento del régimen más concluyente que combina riesgos mínimos para el paciente con altas tasas de éxito terapéutico. Es así que dentro de este estudio hemos demostrado la seguridad y la eficacia de una combinación de terapias para los queloides auriculares y proponemos una posible terapia exitosa.

\section{Correspondencia:}

Karhyn Milagros Ríos-Serna.

Hospital Guillermo Kaelin de la Fuente,

San Borja Norte, Núm. 764, Departamento 801, 15036, Lima-Perú.

Tel: (0051) 945183577

E-mail: karhyn_84@hotmail.com 


\section{BIBLIOGRAFÍA}

1. Arosemena A., Arosemena R. Actualización sobre el queloide y la cicatriz hipertrófica. Act Terap Dermatol. 2007; 30: 238-244.

2. Bejarano SM, Parri FFJ, García SNI, Martínez-Herrada S, Manzanares QA, Albert CA. Cicatrices queloideas en pabellón auricular: un problema no resuelto. Cir Pediatr. 2014; 27 (1): 21-25.

3. Gutiérrez GC, López MF, Lara OJ, Cervantes DJ, Márquez GE, Morales ND. Protocolo de tratamiento de cicatrices queloides en el pabellón auricular del Hospital General Dr. Manuel Gea González. Cir Plást Iberolatinoam. 2012; 38 (1): 49-54.

4. Cuenca-Pardo J, Hernández-Valverde CB, Álvarez-Díaz CJ, Luján-Olivar FS, Escalona-Mancilla A. Tratamiento quirúrgico de cicatrices queloides en oreja. Cir Plast. 2006;16(2):78-83

5. Castillo DP, Troncoso RA, Villamán GJ, Prado SA. Queloides auriculares. Tratamiento quirúrgico y compresivo. Rev Chilena de Cirugía. 2005; 57 (5): 379-383.

6. Poveda JC. La cicatrización queloide. Acta Médica Costarricense. Colegio de Médicos y Cirujanos AMC. 2008; 50 (2): 87-93.

7. Enríquez MJ, Caballero CA. Opciones terapéuticas para cicatrices queloides e hipertróficas. Rev Cent Dermatol Pascua. 2007; 16 (2): 80-84.

8. Small O. Queloide auricular: manejo quirúrgico. Dermatol Perú. 2010; 20 (1): 122-127.
9. Watson D, Panuganti B. Treating scars in the auricle región. Facial Plast Surg Clin North Am. 2016; 25 (1): 73-81.

10. Masoodi Z, Ahmad I, Khurram MF, Haq A. Excision, skin grafting, corticosteroids, adjuvant radiotherapy, pressure therapy, and emancipation: the ESCAPE model for successful taming of giant auricular keloids. Adv Skin Wound Care. 2014; 27 (9): 404-412.

11. Park SY, Lee GH, Park JM, Jin SG, Oh JH. Clinical characteristics of auricular keloids treated with surgical excision. Korean J Audiol. 2012; 16: 134-137.

12. Bran GM, Brom J, Hörmann K, Stuck BA. Auricular keloids combined therapy with a new pressure devise. Arch Facial Plast Surg. 2012; 14 (1): 20-26.

13. Studdiford J, Stonehouse A, Altshuler M, Rinzler E. The management of keloids: hands-on versus hands-off. J Am Board Fam Med. 2008; 21: 149-152.

14. Arredondo MI, Vásquez LA, Arroyave JE, Molina V, Del Río DY, Herrera J. Láser en dermatología. Rev Asoc Colomb Dermatol. 2014; 22 (2): 111-125.

15. Allevato M, Boixeda P. Láser en dermatología. Act Terap Dermatol. 2006; 29 (6): 6-14.

16. Del Pozo J. Láser de $\mathrm{CO} 2$ ablativo en el tratamiento de las cicatrices. Monogr Dermatol. 2016; 29: 153-159. 